\title{
Risk of cancer after long-term therapy of autoimmune disorders with glucocorticoids or DMARDs - a controversial issue
}

\author{
Jakob D. Rudzki
}

Received: 1 February 2019 / Accepted: 20 June 2019 / Published online: 12 July 2019

(C) The Author(s) 2019

\begin{abstract}
Summary Most autoimmune diseases like RA (rheumatoid arthritis) are usually associated with lifelong treatment. The etiology of RA remains unclear, but it depends on a high-risk genetic background and an environmental trigger, which leads to autoimmune dysregulation and results in autoinflammation. This process preferentially affects joints but may spread to different organs and systems. Several studies have illustrated that patients with RA have an increased overall incidence of malignancy compared to the general population (risk of 5-10\%). However, it remains unclear if biological agents like DMARDs (disease-modifying antirheumatic drugs) are associated with a potential increased risk of generating new neoplasms. Data indicate that it is unlikely that RA patients who have received biological agents have a much higher risk of developing lymphoproliferative disorder, other hematologic malignancies or solid tumors compared to MTX (methotrexate) users. In most cases, it also depends on the activity of the underlying rheumatic disease. Thus, clinical decision making must carefully weigh benefits and risks of both aspects of antirheumatic treatment and the risk of elevating the likelihood of developing cancer. The decision to treat with a DMARD must always be based on clinical features and reflect the risk pattern of the individual patient. Up to now, there is no clear evidence that proves the link between longterm DMARD or glucocorticoid use and the increased incidence of newly developed neoplasms. The pathophysiological context is still not well understood and
\end{abstract}

OA Dr. J. D. Rudzki ( $ه)$

Medical University Innsbruck, Innsbruck, Austria

UKIM V-Tirol Kliniken, Landeskrankenhaus Innsbruck, Anichstraße 35, 6020 Innsbruck, Austria

jakob.rudzki@tirol-kliniken.at the underlying link between RA and lymphoma still needs to be addressed.

Keywords Disease-modifying antirheumatic drugs • Methotrexate · Neoplasms · Inflammation · Cancer

bDMARDs and sDMARDs - two big players in treatment of autoimmune diseases

Disease modifying antirheumatic drugs (DMARDs) comprise two large groups of diverse compounds: biological DMARDs (bDMARD) and synthetic DMARDs (sDMARD) (listed in detail in Table 1).

Several studies have presented data that strengthens the association between higher incidence of cancer in RA patients. A nationwide cohort in Taiwan assessed the risk for cancer among 23,644 patients with RA between 1996 and 2007 without any history of malignancies with SIR (standardized incidence rates) of 2.7 for all subtypes of lymphoma (Non-Hodgkin lymphoma [NHL] SIR 3.5; Hodgkin lymphoma [HL] SIR 1.76) [3]. From 2001 to 2005 a Japanese group demonstrated in an observational cohort study an increased SIR for lymphoma in RA patients with an SIR of 6.07 for all types of lymphoma. In this study the potential risk factors were defined as male gender and older age [4]. Setoguchi et al. conducted a cohort study to estimate the effect of biologic DMARDs on cancer. The primary endpoints were hematologic malignancies like multiple myeloma, lymphoma and leukemia as well as common solid cancer like colorectal, lung, stomach, breast, prostate, uterine, ovarian, urinary tract/bladder and finally melanoma. The pooled cohort included 1152 bDMARD users and 7306 MTX users. Comparing the two groups, the propensity score-adjusted pooled hazard ratio was 1.37 (CI 95\% 0.71-2.65) for hematologic malignancies and 0.91 (CI 95\% 0.65-1.26) for solid cancer and revealed 11 hema- 
Table 1 Listing up bDMARDS \& sDMARDs

\begin{tabular}{|c|c|c|c|}
\hline Compound & Trade name ${ }^{\circledR}$ & Description & Administration fequency \\
\hline \multicolumn{4}{|l|}{ bDMARD } \\
\hline Anakinra & Kineret & A human interleukin 1 receptor antagonist protein & SQ daily \\
\hline Infliximab & Remicade & A chimeric (mouse/human) monoclonal anti-TNF-alpha antibody & IV every $4-8$ wk \\
\hline Etanercept & Enbrel & A soluble TNF-alpha receptor fusion protein & SQ weekly \\
\hline Adalimumab & Humira & A fully human anti-TNF-alpha monoclonal antibody & SQ every 2 wk \\
\hline Rituximab & Mabthera, Rituxan & A chimeric (mouse/human) monoclonal anti CD20 directed antibody & IV on $\mathrm{d} 1 / \mathrm{d} 15$, repeated every $24 \mathrm{wk}$ \\
\hline Abatacept & Orencia & $\begin{array}{l}\text { Fusion protein composed of the Fc IgG1 region \& fused to extracellu- } \\
\text { lar domain of CTLA-4 }\end{array}$ & $\begin{array}{l}\text { IV every } 4 \text { wk; } \\
\text { or SQ weekly }\end{array}$ \\
\hline Tocilizumab & Actemra & Humanized anti IL-6 receptor monoclonal antibody & $\begin{array}{l}\text { IV every } 4 \text { wk; } \\
\text { or SQ every } 1-2 \text { wk }\end{array}$ \\
\hline Golimumab & Simponi & A fully human anti-TNF-alpha monoclonal antibody & $\begin{array}{l}\text { IV every } 8 \text { wk; } \\
\text { or SQ every } 4 \text { wk }\end{array}$ \\
\hline Certolizumab-pegol & Cimzia & Fragment of a monoclonal antibody directed against TNF-alpha & SQ, every 2 wk \\
\hline \multicolumn{4}{|l|}{ sDMARD } \\
\hline \multicolumn{4}{|c|}{ Conventional synthetic DMARDs } \\
\hline Methotrexate & Ebetrexat & $\begin{array}{l}\text { Inhibiton of AICAR transformylase } \rightarrow \text { increase of intracellular AMP } \\
\text { and extracellular adenosine }\end{array}$ & Oral, weekly \\
\hline Leflunomide & Arava & $\begin{array}{l}\text { Inhibition of dihydroorotate dehydrogenase, needed for the de novo } \\
\text { synthesis of pyrimidines }\end{array}$ & Oral, daily \\
\hline Hydroxychloroquine & Hydroxychloroquin & Stabilization of lysosomes & Oral, daily \\
\hline Sulfasalazine & Salazopyrin & Immune modulation of unclear mechanism & Oral, daily \\
\hline \multicolumn{2}{|l|}{ Additional sDMARDs: } & \multicolumn{2}{|c|}{$\begin{array}{l}\text { Gold/auranofine, azathioprine, chlorambucil, cyclosporine, cyclophosphamide, mycophenolate, minocycline, } \\
\text { penicillamine, tacrolimus, tofacitinib }\end{array}$} \\
\hline \multicolumn{4}{|c|}{ Targeted synthetic DMARDs } \\
\hline Tofacitinib & Xeljanz & Janus Kinase inhibitor & Oral, daily \\
\hline
\end{tabular}

tologic malignancies and 46 solid tumors during 2940 person-years of bDMARDs use and 88 hematologic malignancies and 558 solid neoplasms during 30,300 person-years of MTX use. The authors concluded that it remains a challenge to study the effect of an infrequent exposure of bDMARDs on rare diseases like hematologic malignancies [5].

Systemic literature review (SLR) of observational studies, including the evidence of the safety of DMARDs and glucocorticoids in patients with rheumatoid arthritis, supported the formation of the recommendations for the management of RA. Patients on tumor necrosis factor inhibitors (TNFi) compared to patients treated with conventional sDMARDs showed a higher risk of dangerous infections (adjusted HR 1.1-1.8), a higher risk of tuberculosis and an increased risk of reactivating infectious diseases like herpes zoster. Interestingly, this group excluded a higher risk for malignancies in general, lymphoma or nonmelanoma skin cancer, except the risk for melanoma, which was described to be elevated (aHR 1.5) [6].

\section{Some compounds in detail}

\section{Methotrexate}

The objective of an Australian study carried out in 2008 focused on assessment of cancer risk of a co- hort of 459 RA patients treated with methotrexate in community practice. Standardized incidence ratios (SIRs) were calculated using state population cancer rates stratified by sex, age (in a 5-year group) and calendar year. Buchbinder et al. estimated a SIR of 1.5 (95\%CI 1.2-1.9) for risk of malignancy among methotrexate-exposed RA patients relative to the general population split up as follows: 3-fold increase in melanoma SIR 3.0 (95\%CI 1.2-6.2), a 5-fold increase in non-Hodgkin's lymphoma SIR 5.1 (95\%CI 2.2-10.0) and finally an almost 3-fold increase in lung cancer SIR 2.9 (95\%CI 1.6-4.8). A consequent and regular skin cancer screening for all RA patients particularly those treated with immunosuppressive agents is highly recommended by the authors [8]. A more recent Swedish study revealed a statistically significant risk increase $(p<0.001)$ in CMM (cutaneous malignant melanoma) observed in MTX-exposed patients compared with patients without MTX exposure. Interestingly, in a subgroup analysis, only women older than the age of 70 years at treatment start preserved the difference between the groups [9]. Hence, this just guides the clinician to take extra care to ensure cancer screening in patients with RA whether methotrexate is prescribed or not.

Solomon et al. carried out a large RA cohort-based study in the US to estimate the comparative risk of cancer across different DMARD groups compared to 
methotrexate, the cornerstone of current RA treatment. Actually, this was the first study done defining MTX as a comparator group. The final study sample comprised 6806 patients, whereas 179 of those patients developed a definite or probable cancer. Finally, the authors concluded that there was no significant increase in cancer risk for the different biologic DMARDs compared with MTX. Rather, they found a reduced risk of cancer among users of TNF antagonists and non-biologic DMARDs users compared with MTX [10].

\section{Tumor necrosis factor inhibitors}

Systemic reviews were performed to estimate the risk ratios (RR) of mortality, cardiovascular events, cancer including hematologic neoplasms like lymphoma but also solid cancer or melanoma and finally even of the association with severe infectious events under treatment with TNFi (tumor necrosis factor inhibitors). As expected primarily serious infections were significantly increased during treatment with TNFi compared to other DMARDs (adjusted HR $[\mathrm{aHR}]=1.1-1.8$ ) [6]. They also confirmed the safety of administration of existing sDMARDs and bDMARDs for the treatment of RA, but DMARDs administration is not without risk. The authors stated that there is a significantly higher risk for upcoming infections like tuberculosis or serious infections of herpes zoster. Finally, Ramiro et al. concluded that the risk of all types of malignancies was not elevated but need to be confirmed and TNFi usage should be considered with reserve [6].

Of particular interest, the administration of TNFi and MTX together as an additional compound compared to MTX alone does not seem to increase the lymphoma risk when using TNFi in the most common way (TNFi plus MTX) (OR 1.1 [95\%CI 0.6-2.0]). Separately analyzed data for infliximab and etanercept reflects similar results and excludes associations between TNFi and lymphoma in a study of lymphomas in 19,562 patients over 89,710 person-years of followup covering anti-TNF exposure in 10,815 patients. The authors concluded that there was no link between an increase in lymphoma with anti-TNF treatment [11].

\section{Glucocorticoids and long-term effects related to elevated risk for malignancies}

Rheumatoid arthritis patients frequently take glucocorticoids as part of their daily regimen. Therefore, glucocorticoids still have a prominent position in the treatment algorithm of RA. However, this is not without fear of their safety profile. During a total duration of less than 2 years of treatment with glucocorticoids, no association was found with lymphoma risk (OR=0.87; 95\%CI 0.51 to 1.5$)$, whereas interestingly treatment over 2 years and beyond was associated to a low lymphoma risk $(\mathrm{OR}=0.43$; 95\%CI $0.26-0.72)$. Importantly, the authors highlighted that the dura- tion of RA disease at the initiation time point of oral steroids did not affect lymphoma risk at all. Intraarticular steroids were linked to a reduced lymphoma risk but only when used as swift flare treatment $(\mathrm{OR}=0.22$ [95\%CI $0.13-0.37])$ and if there is a link to lymphoma then in particular to DLBCL [12]. Additionally, chronic usage of corticosteroids is associated with wound- and bone-healing disorders and surgical site infections [13].

\section{Discussion}

Risk evaluation is difficult because both the autoimmune disease and the chosen treatment option may be associated with an overall increased risk of malignancy or at least some distinct subtypes of malignancies. Interestingly, the overall malignancy risk in patients suffering from RA is quite similar to that of the general population as illustrated in a great metaanalysis published in 2015 [14].

In general, there are some confounders that finally make it impossible to clearly determine whether the potential risk of developing cancer increases after or under the treatment of DMARDs or long-term glucocorticoid usage. There exists a preponderance of evidence that TNFi do not increase the development of most solid tumors with the exception of skin cancer. However, uncertainty remains because of the fact that even recent studies (prospective trials and population-based trials) failed to confirm a strict correlation with increased risk for malignancies [1, 7]. Another confounding factor is defined by increased disease activity that strongly correlates with lymphoma risk [15] especially in case of RA. A group of Korean researchers tried to estimate the incidence of malignancy in early RA patients and to evaluate the relative risk of malignancy by using the Korean National Claims Database comprising a total of 14,081 identified patients. Using a multivariable logistic regression analysis Cho et al. revealed that bDMARDs treatment of early RA patients decreases the overall risk of developing malignancies and this group highlights that it may not affect the risk of developing hematologic malignancies. They also stressed the well-recognized association between RA and malignancies [16].

Other examples for increased risk of specific lymphoma subtypes were primary Sjörgen's syndrome associated with mucosal associated lymphoid tissue (MALT) lymphoma [17] and celiac disease which is linked to enteropathic T cell lymphoma. [18]. DLBCL (diffuse large B cell lymphoma) is reported as the most frequent neoplasm in SLE (systemic lupus erythematodes) which accounts for $37-62 \%$ and is consistent with a role of antigenic drive in autoimmunity-related lymphomagenesis [19]. Regarding the role of immunosuppressive therapy used (cyclophosphamide, azathioprine, methotrexate, mycophenolate mofetil, antimalaria drugs and glucocorticoids), the authors could not find an exclusive explanation. It was possi- 
ble to detect a trend between cyclophosphamide and cumulative steroid use and the appearance of lymphoma disease but without statistical significance. Notably, most of the SLE patients who had lymphoma were free of prior cyclophosphamide therapy [19].

A milestone in understanding the position of DMARDs in the treatment algorithms of rheumatoid arthritis has been the development of registries with well conducted analyses to evaluate safety and effectiveness in direct comparison to RCTs (randomized controlled trials) [6].

Patients suffering from active RA seem to be at a higher risk of lymphoma and lung cancer and a potentially decreased risk for colorectal and breast cancer compared with the general population [1]. Data reported in this paper suggest that a fair estimation of the risk of developing cancer under DMARDs and active RA is not possible. An overall twofold risk was associated with RA and lymphoma compared to general population SIRs 2.08 (95\%CI 1.80-2.39). A considerable higher risk was observed in Hodgkin lymphoma than for non-Hodgkin lymphoma with SIRs of 3.29 (95\%CI 2.56-4.22) and 1.95 (95\%CI 1.7-2.24), respectively [1]. Klein et al. [2, 20] worked out the incidence, pathogenesis and biology of SLE and lymphoma. This review article of 2018 examined the largest and most significant studies published in recent years including all available systemic reviews and meta-analysis as well as large observational cohort studies covering the incidence and risk of lymphoma in SLE. In the end the authors concluded that there are three potential theories to understand the link between SLE and lymphoma. One underlying point involved in lymphomagenesis in SLE bear upon the continuous ongoing lupus activity. Inflammation and autoimmune disease imply an inherent role in triggering chronic immune stimulation which subsequently results in an increased risk for malignancy. Some cytokines and proteins are known to be associated with cell survival and proliferation like BAFF, APRIL, IL-6 and BCL-2 and are also frequently found in both SLE and lymphoma. In contrast to RA, persistent disease activity and SLE could not be linked to development of lymphoma nor was there a robust link to immunosuppressive therapy administered for this disease. Thus, some questions remain regarding the pathogenesis and the link between SLE and lymphoma [20].

\section{Conclusion}

Several crucial questions still remain like what happens to patients with a previous malignancy? Is it still safe to start with bDMARDs in patients with previous malignancies or is it rational or safe to change the bDMARD to a biologic with a different mode of action in case of malignancy occurring during or even after bDMARDs? In this context, registries seem to form a powerful tool to provide relevant information regarding safety concerns. Future trials (randomized clinical trials) addressing those questions may help to gain more relevant information.

Some studies illustrated an increased risk for solid cancer like lung cancer and lymphoma compared to the general population. But there is an unmet need to set up further studies examining specific aspects like lifestyle factors or exposition to toxic substances by smoking to investigate the underlying mechanisms for an increased or decreased risk for cancer in patients with RA or SLE compared to the general population. This way it will be possible to advise and monitor patients accordingly to the generated results [14].

In striking contrast to the mentioned meta-analysis, a French group suggested that by dramatically reducing chronic inflammation in RA patients, TNFi are able to significantly reduce overall mortality and cardiovascular events without increasing the risk of cancer [14].

Despite all limitations an absolute clear statement cannot be given at this point. Only further studies evaluating specific risk factors including RA management strategies, lifestyle factors and the presence or absence of an active inflammatory process might be supportive to shed new light on these open questions.

The administration and treatment with DMARDs are by far safe but some limitations should be considered: age of patients at the start of treatment as well as active inflammatory processes are important factors to assess the risk of future malignancies under or after treatment with DMARDs. Another aspect of promoting opportunistic infections is a critical issue and should be kept in mind during the evaluation process of patients for DMARD therapy approaches.

Funding Open access funding provided by University of Innsbruck and Medical University of Innsbruck.

Conflict of interest J.D Rudzki declares that he has received honoraria and speaker's fee from Gilead-Kite, Novartis, Celgene, BMS, Roche, MSD, AstraZeneca, Amgen and served as an advisor for Gilead-Kite, Novartis, Celgene, BMS, Roche, MSD, AstraZeneca, Amgen.

Open Access This article is distributed under the terms of the Creative Commons Attribution 4.0 International License (http://creativecommons.org/licenses/by/4.0/), which permits unrestricted use, distribution, and reproduction in any medium, provided you give appropriate credit to the original author(s) and the source, provide a link to the Creative Commons license, and indicate if changes were made.

\section{References}

1. Smitten AL, Simon TA, Hochberg MC, Suissa S. A metaanalysis of the incidence of malignancy in adult patients with rheumatoid arthritis. Arthritis Res Ther. 2008;10:R45.

2. Klein A, Polliack A, Gafter-Gvili A. Rheumatoid arthritis and lymphoma: Incidence, pathogenesis, biology, and outcome. Hematol Oncol. 2018;36:733-9.

3. Chen YJ, Chang YT, Wang CB, Wu CY. The risk of cancer in patients with rheumatoid arthritis: A nationwide cohort study in Taiwan. Arthritis Rheum. 2011;63:352-8. 
4. Yamada T, Nakajima A, Inoue E, Tanaka E, Taniguchi A, Momohara S, et al. Incidence of malignancy in Japanese patients with rheumatoid arthritis. Rheumatol Int. 2011;31:1487-92.

5. Setoguchi S, Solomon DH, Weinblatt ME, Katz JN, Avorn J, Glynn RJ, et al. Tumor necrosis factor alpha antagonist use and cancer in patients with rheumatoid arthritis. Arthritis Rheum. 2006;54:2757-64.

6. Ramiro S, Gaujoux-Viala C, Nam JL, Smolen JS, Buch M, Gossec L, et al. Safety of synthetic and biological DMARDs: A systematic literature review informing the 2013 update of the EULAR recommendations for management of rheumatoid arthritis. Ann Rheum Dis. 2014;73:529-35.

7. Thomas E, Symmons DPM, Brewster DH, Black RJ, Macfarlane GJ. National study of cause-specific mortality in rheumatoid arthritis, juvenile chronic arthritis, and other rheumatic conditions: A20 year followup study. J Rheumatol. 2003;30:958-65.

8. Buchbinder R, Barber M, Heuzenroeder L, Wluka AE, Giles G, Hall S, et al. Incidence of melanoma and other malignancies among rheumatoid arthritis patients treated with methotrexate. Arthritis Rheum. 2008;59:794-9.

9. Polesie S, Gillstedt M, Sönnergren HH, Osmancevic A, Paoli J. Methotrexate treatment and risk for cutaneous malignant melanoma: A retrospective comparative registrybased cohort study. Br J Dermatol. 2017;176:1492-9.

10. Solomon DH, Kremer JM, Fisher M, Curtis JR, Furer V, Harrold LR, et al. Comparative cancer risk associated with methotrexate, other non-biologic and biologic diseasemodifying anti-rheumatic drugs. Semin Arthritis Rheum. 2014;43:489-97.

11. Wolfe F, Michaud K. The effect of methotrexate and antitumor necrosis factor therapy on the risk of lymphoma in rheumatoid arthritis in 19,562 patients during 89,710 person-years of observation. Arthritis Rheum. 2007;56:1433-9.

12. Hellgren K, Iliadou A, Rosenquist R, Feltelius N, Backlin C, Enblad G, et al. Rheumatoid arthritis, treatment with corticosteroids and risk of malignant lymphomas: Results from a case-control study. Ann Rheum Dis. 2010;69:654-9.

13. Lamberts SW, Bruining HA, de Jong FH. Corticosteroid therapy in severeillness. NEngl J Med. 1997;337:1285-92.
14. SimonTA, ThompsonA, GandhiKK, Hochberg MC, SuissaS. Incidence of malignancy in adult patients with rheumatoid arthritis: Ameta-analysis. Arthritis Res Ther. 2015;17:212.

15. Ozguler Y, Yazici Y, Hatemi G, Tascilar K, Yazici H. Assessing the possible association of anti-TNF use with new malignancies: A neglected methodological consideration. Pharmacoepidemiol Drug Saf. 2018; https://doi.org/10. $1002 /$ pds.4579.

16. Cho S-K, Lee J, Han M, Bae S-C, Sung Y-K. The risk of malignancy and its incidence in early rheumatoid arthritis patients treated with biologic DMARDs. Arthritis Res Ther. 2017;19:277.

17. Ambrosetti A, Zanotti R, Pattaro C, Lenzi L, Chilosi M, Caramaschi P, et al. Most cases of primary salivary mucosaassociated lymphoid tissuelymphoma are associated either with Sjoegren syndrome or hepatitis $\mathrm{C}$ virus infection. $\mathrm{Br} \mathrm{J}$ Haematol. 2004;126:43-9.

18. Daum S, Wahnschaffe U, Glasenapp R, Borchert M, Ullrich R, Zeitz M, et al. Capsule endoscopy in refractory celiac disease. Endoscopy. 2007;39:455-8.

19. Ekström Smedby K, Vajdic CM, Falster M, Engels EA, Martínez-Maza O, Turner J, et al. Autoimmune disorders and risk of non-Hodgkin lymphoma subtypes: A pooled analysis within the InterLymph Consortium. Blood. 2008;111:4029-38.

20. Klein A, Polliack A, Gafter-Gvili A. Systemic lupus erythematosus and lymphoma: Incidence, pathogenesis and biology. LeukRes. 2018;75:45-9.

Publisher's Note Springer Nature remains neutral with regard to jurisdictional claims in published maps and institutional affiliations.

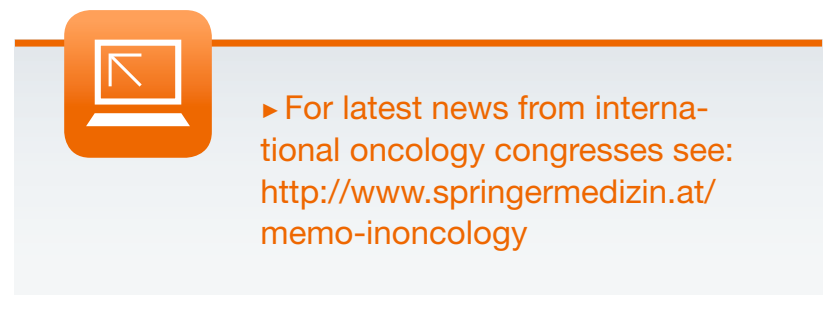

